\title{
Fast, Portable and Low-Cost 3D Foot Digitizers: Validity and Reliability of Measurements
}

\author{
Alfredo BALLESTER*, Ana PIÉROLA, Eduardo PARRILLA, Mateo IZQUIERDO, Jordi URIEL, \\ Beatriz NÁCHER, Vicent ORTIZ, Juan C. GONZÁLEZ, Álvaro F. PAGE, Sandra ALEMANY \\ Instituto de Biomecánica, Universitat Politècnica de València, Valencia, Spain
}

DOI: $10.15221 / 17.218$ http://dx.doi.org/10.15221/17.218
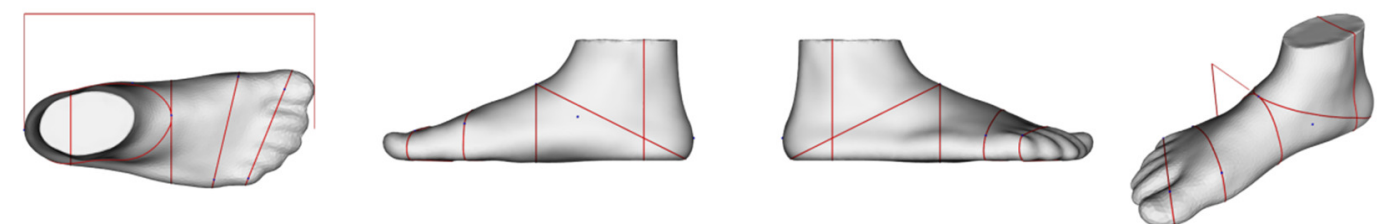

Fig. 1. Examples of digital measurements elicited from a 3D reconstructed foot model using a phone app

\begin{abstract}
This paper presents partial results of a larger validation study of two innovative products based on Data-driven 3D Reconstruction (D3DR) technology: a smartphone app, Avatar3D, and a portable booth, DomeScan. This study quantifies the reliability (Standard Error of Measurement, SEM, Intra-class Correlation Coefficient, ICC) of the automatic foot measurements elicited by these solutions and compares it to traditional anthropometry and to a commercial laser foot scanner. Moreover, it assesses the compatibility (systematic bias) of measurements among these techniques. The results show that, for the six measurements assessed, D3DR is as reliable as high resolution 3D scanners and more reliable than manual measurements made by an expert. Due to its lower cost, speed and portability they could be more suitable for retail and home environments than actual 3D scanners.
\end{abstract}

Keywords: foot scanner, low-cost, data-driven, 3D reconstruction, anthropometry, foot morphology, foot measurements, PCA, accuracy, reliability, SEM, ICC, validity, digital measuring, smartphone app.

\section{Introduction}

Human body shape and dimensions are useful for ergonomic product design $[1,2,3]$. Foot dimensions are required for footwear and foot orthotic design [4,5] as well as for the clinical assessment of foot conditions $[6,7,8,9]$. High accuracy and reliability are indispensable for these purposes.

Historically, the standard method for measuring feet for product design has been traditional anthropometry $[10,11,12,13,14,15]$. This technique requires a trained measurer and specialized metrology instruments such as calipers for linear measurements (heights, lengths and widths) and digital measuring tape for girths. Its accuracy depends on the skills of the measurer and usually there are systematic biases between measurers [16,17]. In clinical environments, radiographic techniques have been commonly used due to their accuracy in taking linear measurements, however it requires more time and resources and the patients are exposed to ionizing radiation [18].

The release of the first commercial 3D foot scanning solutions in the nineties revolutionized the methods for foot measuring [19,20,21]. Foot scanners can acquire the surface of the foot in 3D accurately and enable to measure it digitally [22]. They facilitated the use of the actual foot shapes, and not just measurements, in digital product design [23,24,25]. Since then, different technologies have been developed to gather the actual 3D shape of feet including laser, structured light, stereophotogrammetry [26] and, more recently, depth sensors [27]. Despite their accuracy, they are too bulky and expensive to be used at retail shops or at home.

More recently, and thanks to the databases created using 3D foot scanners, new foot digitizers based on lower-cost methods such as data-driven 3D reconstructions (D3DR) from a few measurements or 2D images had been developed to estimate the 3D shape of faces [28], feet $[29,30,31,32,33,34,35]$ or bodies $[36,37,38,39]$. This technology has much lower hardware requirements than actual $3 D$ scanners, which makes it potentially suitable for being deployed in low-cost simple devices for retail shops or even implemented into a smartphone app, making it possible to and have a foot scanner in your pocket.

*alfredo.ballester@ibv.upv.es; +34 610562 532; http://anthropometry.ibv.org 
In 2015, IBV released two 3D low-cost foot digitizing systems based on D3DR, which have been evolved since then [40]: a smartphone app, Avatar3D*(Fig. 2), and a booth, DomeScan ${ }^{\dagger}$ (Fig. 3).

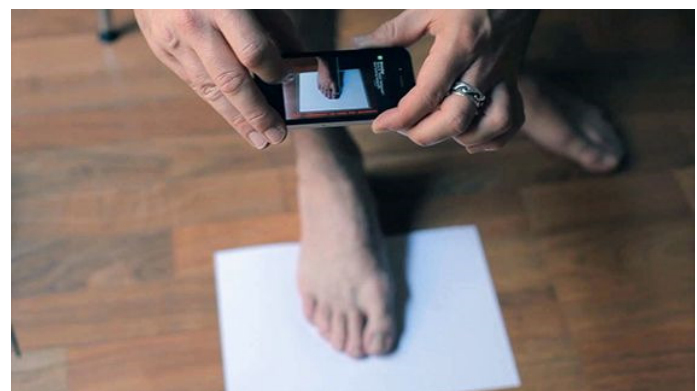

Fig. 2. Avatar3D app

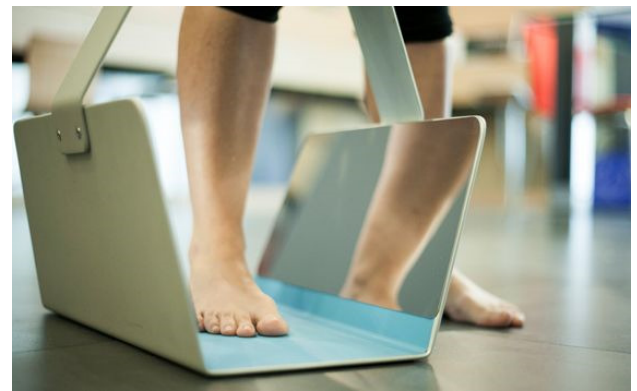

Fig. 3. DomeScan

Avatar3D is a smartphone app that acquires three images (medial, top and lateral, Fig. 4a) of each foot with the rear camera of the smartphone and an A4 or US letter sheet as camera calibration element (Fig. 4a). Images are processed (Fig. 4b) at a remote service that sends back the 3D foot models (Fig. 4c) and a set of measurements in less than 1 minute (Fig. 1). It is conceived to be used at home. A free version of Avatar3D for Android is available on Google Play.

(a)

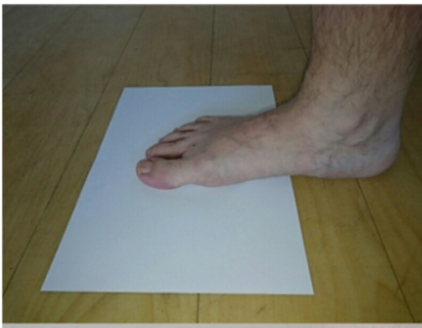

(b)

(c)

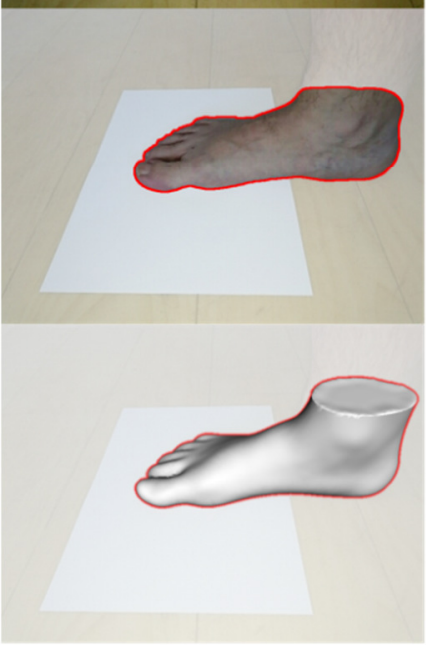

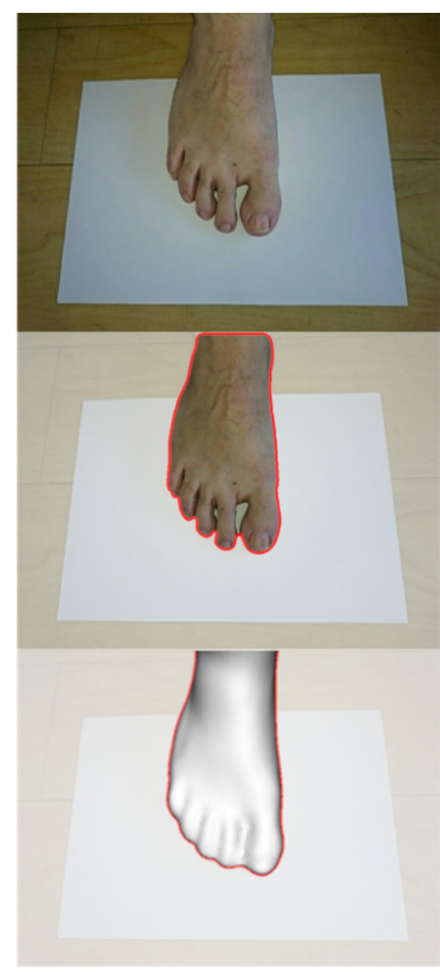

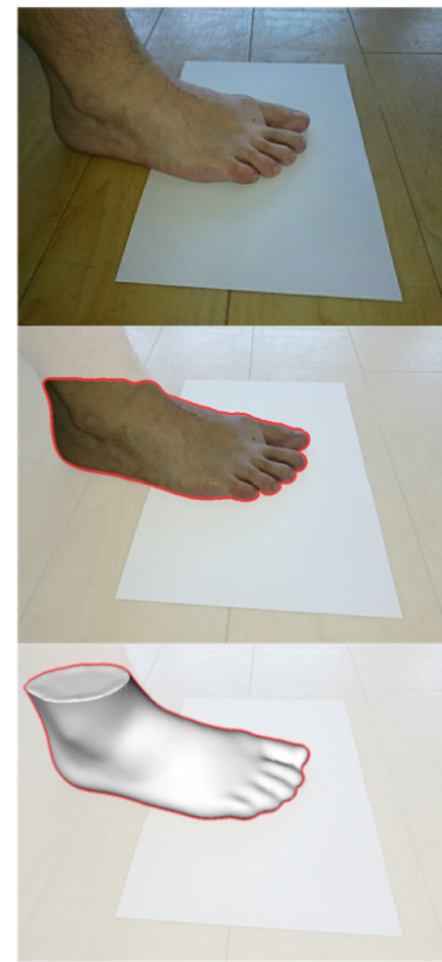

Fig. 4. (a) three foot images (medial, top and lateral) acquired with Avatar3D app,

(b) Segmented foot outlines, and (c) 3D foot matching the three segmented outlines.

DomeScan is a small lightweight booth $(35 \times 45 \times 45 \mathrm{~cm})$ that consists of an aluminum frame in $U$ shape with a non-reflecting vinyl bottom surface and two mirrors on the sides equipped with a Raspberry Pi, camera, Bluetooth communication and illumination system mounted on a bridge over the frame (Fig. 3). DomeScan just needs a single picture that contains the top, medial and lateral images reflected in the mirrors, which makes it the fastest foot scanner in the market. It scans and measures two feet in less than 1 minute. A Windows application running in a PC with Bluetooth connection is used to manage the hardware and the data gathered. DomeScan is conceived for retail shops, labs or scanning points.

\footnotetext{
, Avatar3D: https://www.youtube.com/watch?v=OVni6n1-FS

$\dagger$ DomeScan/IBV: https://www.youtube.com/watch?v=SfP8FHgQ77M
} 
The D3DR process used consists of five steps (Fig. 6): Image Acquisition, Image Segmentation, Camera Calibration, 3D Reconstruction and Digital Measuring. The technology implementation of the first three steps is slightly different for each system because of the differences in hardware (e.g. smartphone vs booth). Foot measuring algorithms are common for both systems. Both use the same space of human foot shape, which was synthesized by applying Principal Components Analysis (PCA) to a database of more than 700 adult foot scans [41] registered to a common parameterization to achieve point-to-point correspondence across feet $[42,34,43]$. This PCA-based foot shape space yields a data-driven method to generate arbitrary foot based on 40 parameters (i.e. the first principal components, Fig. 5).

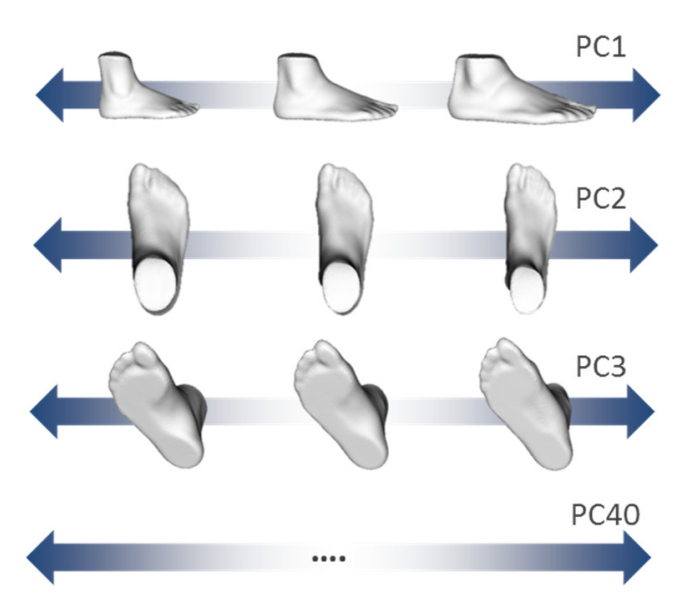

Fig. 5. First 4 Principal Components (PC) of the Foot Shape Space describing different foot shape variations

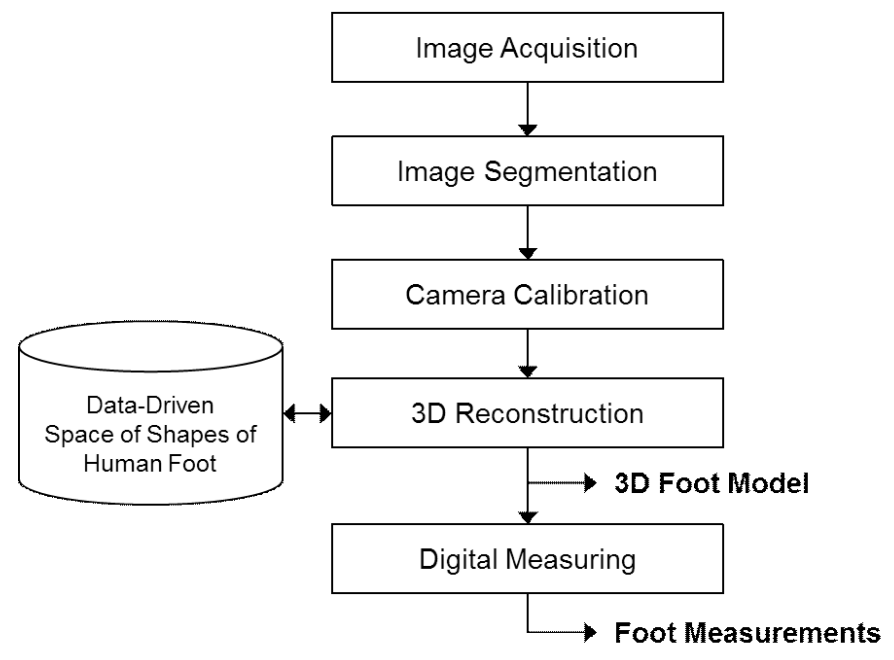

Fig. 6. Data-driven 3D Reconstruction (D3DR) steps

\section{Objectives}

The three objectives of this paper are: (1) to investigate the reliability of measurements extracted from 3D foot models acquired using two implementations of D3DR technology (i.e. a smartphone app, Avatar3D, and a portable booth, DomeScan); (2) to compare them to the established methods, namely traditional anthropometry and actual $3 \mathrm{D}$ foot scanning; and (3) to investigate its validity compared to $3 \mathrm{D}$ foot scanning and traditional anthropometry, both used in product design and clinical practice $[44,45,46,47,48]$.

Based on results, this paper discusses the potential of the technologies assessed and their suitability for different product design applications such as personalization and size selection of footwear and orthotics. This paper focuses only on measurement reliability of healthy foot. The data gathered will also be used in further analyses to quantify the accuracy of D3DR compared to Laser 3D scans, and quantify and compare the reliability of both technologies when scanning human feet.

\section{Materials and methods}

Equipment: Our study aimed to measure bare feet with four techniques: manual measurements using traditional anthropometry, laser 3D scanner, DomeScan, and Avatar3D. Traditional anthropometry measurements were taken by a highly skilled measurer using a scale, a caliper and a measuring tape, with respective precisions of $1,0.1$ and 1 millimeters (Fig. 7). 3D foot scans were taken with Infoot ${ }^{\star}$ (Fig. 8a). Avatar3D prototype app was installed on a Sony Xperia Z (Fig. 8c) and used by a single measurer during the study.

Foot measurements: 22 measurements used in product design and in clinical assessment were considered in our study, from which 6 were selected to be included in this paper due to their relevance. They were Foot Length (FL), Toes Girth (TG), Ball Girth (BG), Ball Width (BW), Instep Girth (IG) and Instep Height $(\mathrm{IH})$. All digital feet gathered digitally in 3D (i.e. with Infoot, DomeScan and Avatar3D) where measured with the same digital measuring algorithms developed by IBV. In contrast to body

\footnotetext{
* INFOOT Scanner, http://www.iwl.jp/main/infoot std.htm
} 
measurements, foot measurements are not standardized. Only FL, BG and BW are considered in ISO 8995 [49], ASTM D5219 [50], ISO 7259 [51], and those definitions are ambiguous and open to different interpretations. Definitions used for the four techniques were those used by the Human Shape Lab of IBV. They comply with standards and are compatible with accepted definitions found in bibliography $[52,53,54,55,56]$.
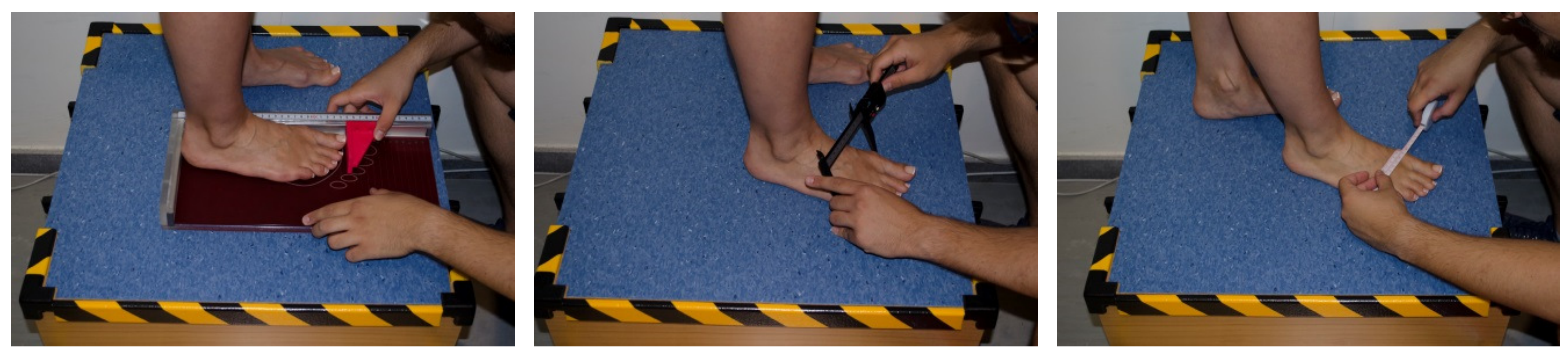

Fig. 7. Traditional anthropometric methods; scale, caliper and measuring tape
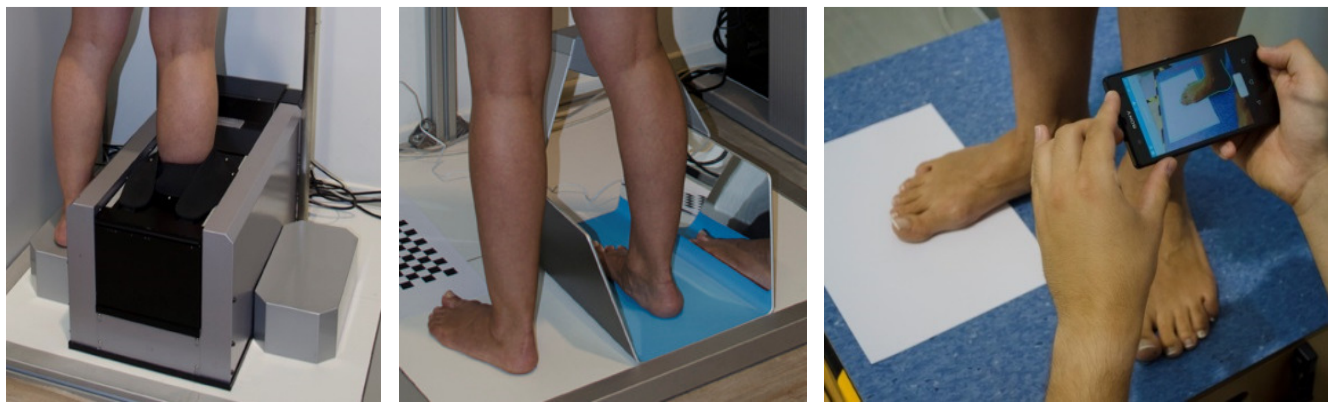

Fig. 8. Digital acquisition of 3D feet, from left to right: Infoot, DomeScan, Avatar3D app.

Design of experiment: 16 healthy adult volunteers (8 females and 8 males) aged 24 to 63 y.o. participated in our study. According to optimal sample size estimation for concurrent designs $[57,58]$, a minimum of 8 subjects is required to assess interrater reliability among 4 instruments with an expected ICC of 0.95 and an acceptable ICC of 0.80 . For intrarater reliability, the minimum number of repetitions with 8 subjects is 3 for expected and acceptable ICCs of 0.99 and 0.95 . Significance and power of the sample size estimations were fixed at $95 \%$ and $80 \%$, respectively. Volunteers were selected to cover the size range between sizes EUR35 (US5w) to EUR47 (US13m). At each measuring session, they were measured three times with each technique in a randomized order while standing comfortably on both feet (half weight bearing, HWB). A total of 192 observations were gathered (16 subjects $x 4$ instruments $\times 3$ repetitions).

Analytical procedures: Reliability was assessed using Standard Error of Measurement (SEM), which provides the magnitude of the random error in measurement units, and Intraclass Correlation Coefficient (ICC), which provides its relative reliability. Validity was assessed by studying the systematic errors of the two new methods (DomeScan and Avatar3D) with respect to the established ones (Infoot 3D scanner and traditional anthropometry), whose validity has been addressed in literature $[17,44,45,46]$. SEM, ICC and systematic errors were calculated according to Elasizw et al. [57] using the function lmer from $R$ package lmerTest for model calculation and significance analysis.

\section{Results}

Reliability: SEM and ICC of the six measurements for each of the four techniques assessed are presented in Table 1 and Table 2. Results show that the four techniques performed similarly. ICCs of measurements from the three digital techniques (Infoot, DomeScan and Avatar3D) were beyond 0.980 and ICCs of traditional anthropometry are beyond 0.970 . SEM of linear measurements (FL, BW, IH) from all techniques were below $1 \mathrm{~mm}$. SEM of measurements from the three digital techniques are below $2 \mathrm{~mm}$ except for TG. SEM of measurements from traditional anthropometry values are below $2 \mathrm{~mm}$ except for TG and BG. 
Validity: Systematic errors in $\mathrm{mm}$ between DomeScan and Avatar3D, and traditional anthropometry and Infoot are presented in Table 3. Results show that the only significant differences between DomeScan and Avatar3D with Infoot are respectively in BW and FL but are just of $1 \mathrm{~mm}$. Significant biases appear between the three digital measuring techniques and the traditional methods, differences in $T G$ and $B G$ are the most significant in value, respectively $4.7-6.1 \mathrm{~mm}$ and $2.3-3.3 \mathrm{~mm}$.

Table 1. SEM values in $\mathrm{mm}$ of the present study for Traditional Anthropometry, Infoot, DomeScan and Avatar3D compared to literature references for Traditional Anthropometry and 3D Foot Digitizers and to Allowable Observer Error (AOE) established by ANSUR study.

\begin{tabular}{cccccccc} 
& Trad. Ant. & Infoot & DomeScan & Avatar3D & $\begin{array}{c}\text { Trad. Ant. } \\
{[16,44,59,60]}\end{array}$ & $\begin{array}{c}\text { 3D Scanner } \\
{[46]}\end{array}$ & AOE [60] \\
\hline FL & 0.9 & 0.8 & 0.6 & 0.7 & $0.5-2.4$ & $0.8-1.0$ & 3 \\
TG & 3.3 & 3.0 & 2.3 & 1.8 & - & - & - \\
BG & 2.1 & 1.6 & 1.2 & 1.0 & $1.8-2.1$ & 1.1 & 4 \\
BW & 0.8 & 0.8 & 0.6 & 0.6 & $1.0-1.8$ & 0.7 & 2 \\
IG & 1.1 & 0.6 & 1.3 & 1.2 & - & 0.6 & - \\
IH & 1.0 & 0.8 & 0.7 & 0.8 & $0.5-1.8$ & $0.5-0.6$ & - \\
\hline
\end{tabular}

Table 2. ICC values of the present study for Traditional Anthropometry, Infoot, DomeScan and Avatar3D compared to literature references for Traditional Anthropometry and 3D Foot Digitizers

\begin{tabular}{ccccccc} 
& & & & Trad. Ant. [5,16, 3D Scanner \\
& Trad. Ant. & Infoot & DomeScan & Avatar3D & $45,61,62,63,64]$ & {$[46,61]$} \\
\hline FL & .998 & .999 & .999 & .999 & $.968-.998$ & $.997-.998$ \\
TG & .977 & .981 & .988 & .993 & - & - \\
BG & .991 & .994 & .997 & .998 & .991 & $.995-.996$ \\
BW & .990 & .992 & .995 & .996 & $.871-.992$ & $.950-.991$ \\
IG & .997 & .999 & .996 & .997 & $.990-.992$ & 0.999 \\
IH & .977 & .985 & .988 & .985 & $.897-.994$ & $.970-.990$ \\
\hline
\end{tabular}

Table 3. Systematic errors in $\mathrm{mm}$ between DomeScan and Avatar3D and traditional anthropometry and Infoot

\begin{tabular}{cccccc} 
& Avatar3D - Infoot & DomeScan - Infoot & Trad.Ant. - Infoot & Trad.Ant. - DomeScan & Trad.Ant. - Avatar3D \\
\hline FL & $1.0^{*}$ & -0.2 & $-0.5^{*}$ & -0.3 & $-1.5^{\star}$ \\
TG & 0.2 & -1.2 & $-5.9^{*}$ & $-4.7^{*}$ & $-6.1^{*}$ \\
BG & -0.1 & -1.0 & $2.3^{*}$ & $3.3^{*}$ & $2.5^{\star}$ \\
BW & 0.1 & $-1.0^{*}$ & $-1.2^{*}$ & -0.2 & $-1.4^{*}$ \\
IG & 0.3 & -0.7 & $-1.1^{*}$ & -0.4 & $-1.3^{*}$ \\
IH & -0.3 & 0.3 & $-2.0^{*}$ & $-2.2^{*}$ & $-1.7^{*}$ \\
\hline
\end{tabular}

* Significant difference $(p<0.05)$

\section{Discussion}

This paper assessed the reliability of four foot measuring techniques and compared them. SEM and ICC obtained for Infoot and traditional anthropometry measurements are consistent with prior literature $[5,16,44,46,59,60,61,62,63,64]$, cf. Table 1 and Table 2. ICC values for the four techniques and the six measurements showed excellent reliability (beyond 0.95). SEM values for all techniques are within the allowable intra-observer error established by ANSUR [60]. Avatar3D and DomeScan are as reliable as Infoot for FL, BW and IH and slightly more reliable than Infoot for TG and BG. Infoot is more reliable for IG. TG is the least reliable of all measurements possibly because toe area is the most articulated part of the foot and toes can adopt slightly different poses at each repetition [65]; moreover, uncertainty in the location of the small joints (toes) may also have an influence [66]. These errors correspond to within-rater (intra-observer) variability; namely, a single scanner unit in the case of DomeScan and Infoot and a single measurer in the case of Avatar3D app and traditional anthropometry. It has to be noted that in traditional anthropometry, inter-observer errors are greater than intra-observer ones $[17,45,66,67]$.

This paper also assessed the validity of two innovative products based on Data-driven 3D Reconstruction (D3DR) technology: a smartphone app, Avatar3D, and a portable booth, DomeScan. Measurements elicited from 3D feet acquired with D3DR technologies were compared to those of Infoot scanner and traditional anthropometry. Systematic error of measurements from D3DR technologies compared to Infoot is small (1 $1 \mathrm{~mm}$ or less) and not significant. Both DomeScan and 
Avatar3D measurements can be considered compatible with Infoot measurements according to ISO 20685 [68], which establishes a threshold of $2 \mathrm{~mm}$ for foot dimensions [17].

Systematic errors found between the three digital techniques and the traditional anthropometry are consistent with prior literature $[5,45,46]$. Traditional anthropometry compared to digital techniques underestimated all measurements except BG. In our study, these systematic differences could be due to the pressure exerted over the soft tissue during manual measuring (touching vs. non-touching measurement), to slight differences in the measuring posture/weight-bearing with traditional methods and to systematic differences between the palpated landmarks of traditional anthropometry and the geometrically searched landmarks [47,69]. These systematic biases have been reported to be adjustable by linear correction, if required $[45,46]$.

The accuracy and reliability of the digital techniques seem adequate for both product design (footwear and orthotics) and clinical assessment. The main advantage of D3DR implementations compared to actual 3D scanners is its efficiency, which results in lower costs and fastest processing because they do not require high-end cameras, projectors and computing resources. Avatar3D app and DomeScan are, respectively, the most portable and the fastest 3D foot scanning solutions available.

The validation of the full list of measurements and of the 3D models obtained by D3DR techniques, not investigated in this study, is however necessary. Moreover, in order to fully assess adequacy of these techniques (along with other 3D scanning solutions) for such uses, it would also be necessary to quantify the effects of different factors affecting significantly bare foot measurements methods such as posture/weight-bearing (fixed effect), foot swelling along the day (fixed effect) and the uncertainty related of measuring the same person on different days (random effect).

\section{Conclusions}

The results show that, for the six measurements assessed in healthy adults, D3DR is as reliable as high resolution 3D scanners and slightly more reliable than manual measurements made by one expert. Due to its lower cost, speed and portability they have the potential to be more suitable than actual 3D scanners in certain contexts. Avatar3D app can be massively distributed and comfortably used at home for online size recommendations or for made-to-measure (MtM) orders of footwear, insoles and orthotics and even at general medical practitioner offices. DomeScan can be used at points with high measuring traffic such as retail shops.

\section{Acknowledgements}

The work described in this paper has been partially supported by the Valencia Region Government (IVACE) and FEDER funds under the research project CUSTOM_ON_BODY (IMDECA/2016/11 and IMDEEA/2017/57). The authors would like to thank their colleagues José Montero, Francisco Roldán, Julio A. Vivas, Jesús Selles, Silvia San Jerónimo, Marian Candel and Pablo Iscar for the implementation of Avatar3D and DomeScan systems; Ana B. Ferrer for her contribution to the conduction of tests; and Carlos Soler and Miguel A. Artacho for fostering the conduction of this work.

\section{References}

[1] Pheasant, S., (1988). Bodyspace: Anthropometry, Ergonomics and Design. Taylor and Francis, NY, Philadelphia.

[2] Guidelines for using anthropometric data in product design, HFES 300 Commitee, 2004.

[3] Robinette, K. M., (2012) Anthropometry for product design, In Handbook of Human Factors and Ergomomics 4.

[4] Xiong S., (2013) Foot models and measurements, in Handbook of footwear Design and Manufacture, Woodhead Publishing, Ed. By Luximon, A.

[5] Laughton, C. et al. (2002). A comparison of four methods of obtaining a negative impression of the foot. JAPMA, 92(5), 261-268.

[6] Payne, C. B. (1998). Biomechanics of the foot in diabetes mellitus. Some theoretical considerations. JAPMA, 88(6), 285-289.

[7] Williams, D. S. et al. (2001). Arch structure and injury patterns in runners. Clinical biomechanics, 16(4), 341-347.

[8] Franettovich, M. M. et al. (2007). The ability to predict dynamic foot posture from static measurements. JAPMA, 97(2), 115-120.

[9] Friends, J. et al. (2008). A comparison of different assessment techniques for measuring foot and ankle volume in healthy adults. JAPMA, 98(2), 85-94. 
[10] Freedman, A. et al. (1946). Foot dimensions of soldiers. Third Partial Report Project NT-13. Fort Knox, AMR Laboratory.

[11] Rossi, W.A., (1983). The high incidence of mismated feet in population. Foot and Ankle 4, 105-112.

[12] Hawes, M.R. and Sovak, D., (1994). Quantitative morphology of the human foot in a North American population. Ergonomics 37 (7), 1213-1226.

[13] Kouchi, M., (2003). Inter-generation differences in foot morphology: aging or secular change? J of H Ergology 32, 23-48.

[14] Bunch, R.P., (1988). Foot measurement strategies for fitting athletes. J of Testing and Evaluation 16 (4), 407-411

[15] Wehr, A., (1999). 3-Imaging laser scanner for close range metrology. In: Proc. of the SPIE Conf on Laser Radar Technol. and Applicat. IV, Orlando, FL, SPIE, vol. 3707, pp. 381-389.

[16] Liu, W. et al. (1999). Accuracy and reliability of a technique for quantifying foot shape, dimensions and structural characteristics. Ergonomics 42 (2), 346-358.

[17] Kouchi, M. et al. (2014). Anthropometric methods for apparel design: Body measurement devices and techniques. In: Anthropometry, apparel sizing and design, Woodhead Publishing, 67-94.

[18] Mall, N. A. et al. (2007). The reliability and reproducibility of foot type measurements using a mirrored foot photo box and digital photography compared to caliper measurements. Journal of biomechanics, 40(5), 1171-1176.

[19] Blais, F. et al. (2000). The shape grabber footscanner: a low cost high accuracy 3-D system for the acquisition of human feet. In: Corner, B.D., Nurre, J.H. (Eds.), Three-Dimensional Image Capture and Applications, vol. III. Proc of the SPIE, vol. 3958, pp. 178-186.

[20] Gartner, H. et al. (1999). Multiple structured light system for the 3D-measurement of feet. In: Proc of Three-dimensional Image Capture and Applications II (ISandT/ SPIE) Conf, San Jose, CA, SPIE, vol, 3640, pp. 104-114.

[21] Houle, P.S. et al. (1997). Novel fully integrated computer system for custom footwear: from 3D digitization to manufacturing. Proc of Three-dimensional Image Capture and Apps, SPIE, vol. 3313, San Jose, CA, pp. 65-73.

[22] Liu, X. et al. (2004). 3D characterization and localization of anatomical landmarks of the foot by FastSCAN. Real-time imaging, 10(4), 217-228.

[23] Treleaven P, (2004). Sizing us up - new 3-d body scanners are reshaping clothing car seats and more. IEEE Spectrum 2004, 41:17-19.

[24] Duffy, V. G. (2007). Modified virtual build methodology for computer-aided ergonomics and safety. Human Factors and Ergonomics in Manufacturing and Service Industries, 17(5), 413-422.

[25] Rajulu, S. and Corner, B.D., (2012) 3D Surface Scanning, in Science of Footwear, CRC Press, Ed. By Goonetileke, R.S.

[26] Saunders, C. G. and Chang, J. (2012) 3D Shape Capture of Human Feet and Shoe Lasts, in Science of Footwear, CRC Press, Ed. By Goonetileke, R. S.

[27] Lovato, C. et al. (2014). A low cost and easy to use setup for foot scanning. In Proc of the 5th Int Conf on 3D Body Scanning Technologies, Lugano, Switzerland (pp. 21-22).

[28] Blanz, V. and Vetter, T. (1999, July). A morphable model for the synthesis of 3D faces. In Proc of the 26th annual conf on Computer graphics and interactive techniques (pp. 187-194). ACM Press/Addison-Wesley Publishing Co.

[29] Luximon, A. and Goonetilleke, R. S. (2004). Foot shape modeling. Human Factors, 46(2), 304-315.

[30] Wang, J., Saito, H., Kimura, M., Mochimaru, M., and Kanade, T. (2004). Reconstruction of human foot from multiple camera images with 3D active contour models. AlSM'04 Proc, 390-395.

[31] Wang, J. et al. (2005, June). Shape reconstruction of human foot from multi-camera images based on PCA of human shape database. In Proc of 5th Int Conf on 3-D Digital Imaging and Modeling, pp. 424-431, IEEE.

[32] Wang, J. et al. (2006). Human foot reconstruction from multiple camera images with foot shape database. IEICE Trans on Information and Systems, 89(5), 1732-1742.

[33] Amstutz, E. et al. (2008). PCA-based 3D shape reconstruction of human foot using multiple viewpoint cameras. Int $J$ of Automation and Computing, 5(3), 217-225.

[34] Ma, X., and Luximon, A. (2014). 3D foot prediction method for low cost scanning. Int J of Indl Erg, 44(6), 866-873.

[35] Parrilla, E. et al. (2015), "Low-cost 3D foot scanner using a mobile app", Footwear Science, Vol. 7, Iss. sup1.

[36] Seo, H., Yeo, Y. I., and Wohn, K. (2006, April). 3D body reconstruction from photos based on range scan. In Int Conf on Technologies for E-Learning and Digital Entertainment (pp. 849-860). Springer, Berlin, Heidelberg.

[37] Boisvert, J. et al. (2013). Three-dimensional human shape inference from silhouettes: reconstruction and validation. Machine vision and applications, 24(1), 145-157.

[38] Zhu, S. et al. (2013). An efficient human model customization method based on orthogonal-view monocular photos. Computer-Aided Design, 45(11), 1314-1332. 
[39] Ballester, A. et al. (2016). Data-driven three-dimensional reconstruction of human bodies using a mobile phone app. Int J of the Digital Human, 1(4), 361-388.

[40] González, J.C., Making 3D foot scanning technology possible from any smartphone, Linkedln Pulse, J17

[41] García-Hernández, J. et al. (2005). The MORFO3D foot database. In Iberian Conf on Pattern Recognition and Image Analysis (pp. 658-665). Springer, Berlin, Heidelberg.

[42] Allen, B. et al. (2003). The space of human body shapes: reconstruction and parameterization from range scans, in ACM ToG, Vol. 22, No. 3, pp. 587-594.

[43] Ballester, A. et al. (2015). Low-Cost Data-Driven 3D Reconstruction and its Applications, In Proc. of 6th Int. Conf. on 3D Body Scanning Technologies, Lugano, Switzerland, October 2015. doi:10.15221/15.184

[44] Williams, D. S., and McClay, I. S. (2000). Measurements used to characterize the foot and the medial longitudinal arch: reliability and validity. Physical therapy, 80(9), 864-871.

[45] Witana, C. P. et al. (2006). Foot measurements from three-dimensional scans: A comparison and evaluation of different methods. Int J of Industrial Ergonomics, 36(9), 789-807.

[46] De Mits, S. et al. (2010). Reliability and validity of the Infoot 3D foot digitizer for normal healthy adults. Footwear Science, 2(2), 65-75.

[47] Telfer, S., and Woodburn, J. (2010). The use of 3D surface scanning for the measurement and assessment of the human foot. Journal of foot and ankle research, 3(1), 19.

[48] De Mits, S. et al. (2011). Reliability and validity of the INFOOT three-dimensional foot digitizer for patients with rheumatoid arthritis. JAPMA, 101(3), 198-207

[49] ISO 8559-1:2017 Size designation of clothes -- Part 1: Anthropometric definitions for body measurement

[50] ASTM D5219-15 Standard Terminology Relating to Body Dimensions for Apparel Sizing

[51] ISO 7250-1:2008 Basic human body measurements for technological design -- Part 1

[52] Rossi, W. A. and Tennant, R. (1993). Professional shoe fitting. National Shoe Retailers Association.

[53] Ramiro, J. et al. (1994). Guía de recomendaciones para el diseño de calzado. Instituto de Biomecánica de Valencia.

[54] AIST, Digital Human Research Group, http://www.dh.aist.go.jp/research/centered/anthropometry/M_foot.html.en

[55] Goonetilleke, R. S. (Ed.). (2012). The science of footwear. CRC Press.

[56] Luximon, A. (Ed.). (2013). Handbook of footwear design and manufacture. Elsevier.

[57] Eliasziw, M. et al. (1994). Statistical methodology for the concurrent assessment of interrater and intrarater reliability: using goniometric measurements as an example. Physical therapy, 74(8), 777-788.

[58] Walter, S. D. et al. (1998). Sample size and optimal designs for reliability studies. Statistics in medicine, 17(1), 101-110.

[59] Kouchi, M. et al. (1996). Random errors in anthropometry. Journal of human ergology, 25(2), 155-166.

[60] Gordon C. C. et al. (1989). 1987-1988 Anthropometric Survey of U.S. Army Personnel: Methods and Summary Statistics, Technical Report NATICK/TR-89-004. Natick, MA: U.S. Army Natick Research, Devand Eng Center.

[61] Lee, Y. C. et al. (2014). Comparing 3D foot scanning with conventional measurement methods. Journal of foot and ankle research, 7(1), 44.

[62] Butler, R. J. et al. (2008). Arch height index measurement system: establishment of reliability and normative values. JAPMA, 98(2), 102-106.

[63] Pohl, M. B. and Farr, L. (2010). A comparison of foot arch measurement reliability using both digital photography and calliper methods. Journal of foot and ankle research, 3(1), 14.

[64] Mall, N. A. et al. (2005). The reliability and reproducibility of foot measurements using a mirrored foot photo box compared to calliper measurements. In American Society of Biomechanics National Meeting, Cleveland, $\mathrm{OH}$.

[65] Corner B.D. and Hu A. (1998). The effect of sway on image fidelity in whole body digitizing. In J Nurre and BD Corner (eds.), Three-Dimensional Image Capture and Applications, Seattle, WA. Proc of ISandT/SPIE Sympon Electronic Imaging: Science and Technology, San Jose, CA, Vol. 3227, pp. 36-45.

[66] Kouchi, M., and Mochimaru, M. (2011). Errors in landmarking and the evaluation of the accuracy of traditional and 3D anthropometry. Applied ergonomics, 42(3), 518-527.

[67] Kouchi, M. et al. (1999). Interobserver errors in anthropometry. Journal of human ergology, 28(1-2), 15-24.

[68] ISO 20685:2010 3-D scanning methodologies for internationally compatible anthropometric databases

[69] Kouchi, M., and Mochimaru, M. (2006). Why Scan-Derived Body Measurements Differ From Measurements by the Traditional Methods. In Proceedings IEA 2006 Congress. 